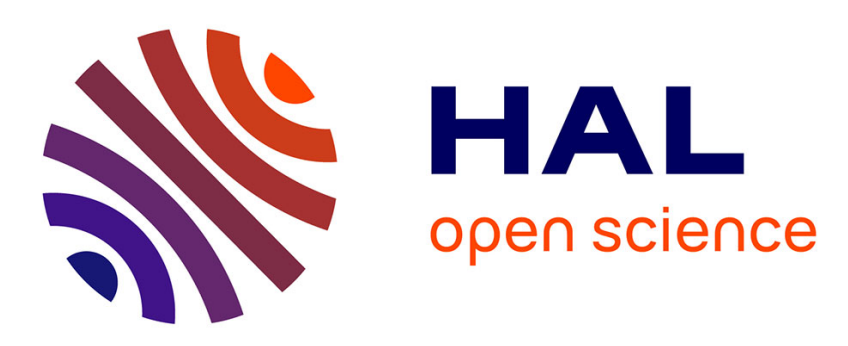

\title{
Alkali atoms attached to vortex-hosting helium nanodroplets
}

Ernesto García-Alfonso, François Coppens, Manuel Barranco, Martí Pi, Frank Stienkemeier, Nadine Halberstadt

\section{- To cite this version:}

Ernesto García-Alfonso, François Coppens, Manuel Barranco, Martí Pi, Frank Stienkemeier, et al.. Alkali atoms attached to vortex-hosting helium nanodroplets. Journal of Chemical Physics, 2020, 152 (19), 10.1063/5.0008923 . hal-03086640

\section{HAL Id: hal-03086640 https://hal.science/hal-03086640}

Submitted on 22 Dec 2020

HAL is a multi-disciplinary open access archive for the deposit and dissemination of scientific research documents, whether they are published or not. The documents may come from teaching and research institutions in France or abroad, or from public or private research centers.
L'archive ouverte pluridisciplinaire $\mathbf{H A L}$, est destinée au dépôt et à la diffusion de documents scientifiques de niveau recherche, publiés ou non, émanant des établissements d'enseignement et de recherche français ou étrangers, des laboratoires publics ou privés. 


\title{
Alkali atoms attached to vortex-hosting Helium nanodroplets
}

\author{
Ernesto García-Alfonso, ${ }^{1,2}$ Francois Coppens, ${ }^{2}$ Manuel Barranco, ${ }^{2,3,4}$ \\ Martí $\mathrm{Pi},{ }^{3,4}, *$ Frank Stienkemeier, ${ }^{5, \dagger}$ and Nadine Halberstadt ${ }^{2, \ddagger}$ \\ ${ }^{1}$ Facultad de Física, Universidad de La Habana, 10400 La Habana, Cuba \\ ${ }^{2}$ Université Toulouse 3 and CNRS, Laboratoire des Collisions, Agrégats et Réactivité, \\ IRSAMC, 118 route de Narbonne, F-31062 Toulouse Cedex 09, France \\ ${ }^{3}$ Departament FQA, Facultat de Física, Universitat de Barcelona. Diagonal 645, 08028 Barcelona, Spain \\ ${ }^{4}$ Institute of Nanoscience and Nanotechnology (IN2UB), Universitat de Barcelona, Barcelona, Spain. \\ ${ }^{5}$ Physikalisches Institut, Universität Freiburg, 79104 Freiburg, Germany.
}

(Dated: May 1, 2020)

\begin{abstract}
Light absorption or accordingly fluorescence excitation spectroscopy of alkali atoms attached to ${ }^{4} \mathrm{He}$ droplets is investigated as a possible way of detecting the presence of vortices. To this end, we have calculated the equilibrium configuration and energetics of alkali atoms attached to a ${ }^{4} \mathrm{He}_{1000}$ droplet hosting a vortex line using ${ }^{4} \mathrm{He}$ density functional theory. We use them to study how the dipole absorption spectrum of the alkali atom is modified when the impurity is attached to a vortex line. Spectra are found to be shifted to the blue (higher frequencies) and broadened compared to vortex-free droplets, because the dimple in which the alkali atom sits at the intersection of the vortex line and the droplet surface is deeper. This effect is smaller for lighter alkali atoms, and all the more so when using a quantum description since in this case, they sit further away from the droplet surface on average due to their zero-point motion. Spectral modifications due to the presence of a vortex line are minor for $n p \leftarrow n s$ excitation, and therefore insufficient for vortex detection. In the case of higher $n^{\prime} p \leftarrow n s$ or $n^{\prime} s \leftarrow n s\left(n^{\prime}>n\right)$ excitations the shifts are larger, as the excited state orbital is more extended and therefore more sensitive to changes in the surrounding helium density.
\end{abstract}

\section{INTRODUCTION}

${ }^{4} \mathrm{He}$ droplets built in supersonic beam experiments can be characterized by a tempe@ture of about $0.37 \mathrm{~K}$.[1] At that temperature they are superfluid, which makes them ideal ultra-cold matrices for spectroscopy studies of captured dopants. They are also ideal laboratories for addressing superfluidity at the nanoscale.[2-6] In particular, they may host quantized vortices, a dramatic fingerprint offthe superfluid state.[7-9] Since their existence was proposed by Onsager and Feynman, the study and detection of quantized vortices in superfluid and superconducting systems has not ceased and attracts the interest of physicists and chemists working in low temperature processes, semiconductors, quantum optics, and atomic and molecular physics and chemistry. It is hard to find a more widespread subject of study.

Quantized vortices are ubiquitous in quantum systems under rotation, as e.g. liquid helium samples at temperatures below $2.17 \mathrm{~K}$ (the superfluid transition temperature),[7] cold-gas Bose-Einstein condensates, [10] and electron droplets in low-dimensional quantum dots in a magnetic field.[11] However, the only small selfbound system where vortices are likely to occur is a ${ }^{4} \mathrm{He}$ droplet. $[12,13]$

So far, no clear experimental signature of vortex formation in small ${ }^{4} \mathrm{He}$ droplets has been found, although this

*Electronic address: marti@fqa.ub.edu

'Electronic address: st enkemeier@uni-freiburg.de

+Electronic address: Nadine.Halberstadt@irsamc.ups-tlse.fr has been the subject of several experimental and theoretical proposals and studies. Many of them involve light absorption or laser induced fluorescence excitation spectroscopy, which usually give similar spectra, the former being easier to simulate theoretically and the latter easier to measure experimentally: we will refer to them as light absorption spectroscopy, or simply absorption spectroscopy, throughout this article. Close et al.[14] suggested the light absorption spectrum of $\mathrm{Ag}$ atoms in the bulk of superfluid droplets as a possible candidate: if $\mathrm{Ag}$ atoms are attached to the vortex line, their environment changes and this could be reflected in the absorption spectrum. To our knowledge, this possibility has never been explored. Along the same line, a theoretical study has been carried out for electron bubbles attached to vortex lines in superfluid liquid helium as a function of pressure. It was shown that the electron absorption spectrum is shifted by the presence of a vortex line, but the shift is too small to be experimentally detectable.[15]

Another theoretical proposal,[16] which has not been tested experimentally either, suggested that microwave absorption spectroscopy of a Ca dopant could show a difference when a vortex was present. More recently, absorption spectros opy was also suggested[17] for the same dopant. The rationale is basically the same as for $\mathrm{Ag}$ atoms: the change induced in the dopant environment due to its binding to a vortex line would translate as a change in the absorption spectrum. Ca impurities are known to reside in a deep dimple at the surface of the helium droplet.[17] Their binding to a vortex line would result in their further sinking inside the droplet. As a result, their absorption spectrum would evolve from that of an impurity on the droplet surface to that of an im- 
purity in its bulk. Hence a satellite peak should appear, which would be blue-shifted from the nain peak and with an intensity depending on the number of vortex-hosting droplets. Inspection of existing experimental results[18] showed no such signature.

It is worth stressing that these proposals and experiments involve droplets made of several thousand $\mathrm{He}$ atoms created by adiabatic expansion of a helium gas into vacuum.[1] All attempts to detect the presence of vortices in droplets of this size have failed so far. Very recently, large ${ }^{4} \mathrm{He}$ droplets made of $10^{8}-10^{11}$ atoms have been created using hydrodynamic instability of a cryogenic liquid helium jet passing through the nozzle of a molecular beam apparatus,, as reviewed in Refs. 9 and 19. Helium drops, which are initially produced in the normal, non-superfluid phase, can acquire angular momentum during the passage of the fluid through the nozzle. Such droplets could be analyzed as single objects by x-ray and XUV light using intense high harmonics sources. [20, 21] This has made it possible to determine their shapes and, doping them with Xe atoms, [20, 22] the presence of vortices, confirming their superfluid nature.

The following question therefore arises: why have vortices not been detected in smaller droplets $\left(10^{3}-10^{4}\right.$ atoms)? Is it because no clear signature of their presence has been found yet? Or is it because vortices are too scarce due to the droplet formation and doping mechanisms? Let us recall that droplets containing a few thousand He atoms are produced in subcritical conditions by atom condensation and evaporative cooling, [1] which does not favor deposition of large amounts of angular momentum in the droplet. Some angular momentum is transferred to the droplet during the impurity pickup, which does nucleate vortex rings and loops. [23-26] However, they are dynamically unstable. At variance, large droplets such as those detected as single objects using x-ray or XUV light are created by hydrodynamical instabilities of a very cold but non-superfluid He jet. They can acquire a large amount of angular momentum during their passage through the nozzle of the experimental apparatus. When the droplets become superfluid, this angular momentum helps nucleate vortex arrays stable enough to be expermentally detected.[20, 22]

Since light absorption spectroscopy is very sensitive to the impurity environment, it is a good candidate as a tool for detecting impurities attached to vortices. We have therefore calculated the absorption spectrum of alkali $(\mathrm{Ak})$ atoms from $\mathrm{Li}$ to $\mathrm{Cs}$ when attached to a vortex line in a superfluid droplet, and compared to the same spectrum with no vortex. The goal is to provide a quantitative answer to the size of the shift that can be expected, and therefore to the possibility for photon absorption experiments to detect the presence of vortices. The absorption spectrum of alkali atoms attached to He droplets has been thoroughly studied experimentally in the past, see e.g. Refs. 27-32, and earlier works have simulated photon absorption spectra of alkali atoms attached to vortex-free helium nanodroplets using different functionals and/or Hela kali pair potentials. [27, 28, 3337] We have repeated them in order to ensure comparison with vortex-hosting droplets with exactly the same parameters. To the best of our knowledge, the spectrum of alkali atoms attached to vortex-hosting droplets has not been previously addressed theoretically.

This article is organized as follows. In Sec. II we outline the ${ }^{4} \mathrm{He}$ Density Functional Theory $\left({ }^{4} \mathrm{He}-\mathrm{DFT}\right)$ approach used to simulate doped ${ }^{4} \mathrm{He}$ droplets and their photon absorption spectrum. The results are presented and discussed in Sec. III, and a Summary and Outlook are given in Sec. IV.

\section{THE ${ }^{4}$ HE-DFT APPROACH}

We have used the ${ }^{4} \mathrm{He}-\mathrm{DFT}$ method to describe superfluid helium droplets. This approach has been recently reviewed and comprehensively described in Ref. 35 , where the interested reader may find all the details for its application to pure and doped ${ }^{4} \mathrm{He}$ drops hosting or not vortex lines. We only give here the main features and some details to help following the discussion of the results.

Within ${ }^{4} \mathrm{He}-\mathrm{DFT}$, the total energy of a ${ }^{4} \mathrm{He}_{N}$ droplet at zero temperature is written as a functional of the ${ }^{4} \mathrm{He}$ atomic density $\rho(\mathbf{r})$

$$
E[\rho]=T[\rho]+E_{c}[\rho]=\frac{\hbar^{2}}{2 m} \int d \mathbf{r}|\nabla \Psi(\mathbf{r})|^{2}+\int d \mathbf{r} \mathcal{E}_{c}[\rho]
$$

where $T[\rho]$ is the kinetic energy, $\Psi(\mathbf{r})$ an effective wave function defined by $\rho(\mathbf{r})=|\Psi(\mathbf{r})|^{2}$, and the functional $\mathcal{E}_{c}$ contains the He-He interaction term (within Hartree approximation) as well as additional terms describing nonlocal correlation effects. Strictu sensu, for vortex-free droplets $\Psi(\mathbf{r})$ is a real function and hence $\Psi(\mathbf{r})=\sqrt{\rho(\mathbf{r})}$

The results presented in this work have been obtained using the most accurate He functional to date, namely the Orsay-Trento functional (OT).[38] It includes a nonlocal correction to the kinetic energy and a backflow contribution. The backflow term only plays a role in the presence of density currents, which is the case in vortex-hosting droplets. Both contributions (especially the backflow term) make the OT functional computationally quite expensive. In addition to the computational cost, numerical problems arise in the case of helium samples doped with very attractive impurities. [35] Several variants of the OT functional have been designed and used to circumvent this problem. The so-called "solid functional" [39] has become very popular in static and time-dependent applications because it contains a "penalty term" to prevent the He density to become unphysically large when the helium sample is doped with very attractive impurities. The results obtained with this simplified functional are presented in the Supplementary Material. Because the backflow term should be the main difference between both functionals (the alkali-helium in- 
teraction is only weakly attractive), comparing the results in the Supplementary Material with those presented here will give an idea of the importance of this term for absorption spectra in the presence of a vortex.

For alkali-doped droplets Eq. (1) has to be modified to include the interaction of the droplet with the dopant. For the heavier alkalis, $\mathrm{Ak} \equiv \mathrm{K}, \mathrm{Rb}$ or $\mathrm{Cs}$, which can be described as point-like, classical particles, this is simply done by adding the impurity-liquid interaction energy to the energy functional $E[\rho]$. This term, which acts as an external field for the helium density, is constructed within the pairwise sum approximation by integrating the impurity-He atom interaction, $V_{A k}$, over the liquid density,

$$
E[\rho] \rightarrow E[\rho]+\int d \mathbf{r} \rho(\mathbf{r}) V_{A k}\left(\left|\mathbf{r}-\mathbf{r}_{A k}\right|\right)
$$

where $\mathbf{r}_{A k}$ is the location of the impurity.

The droplet equilibrium configuration is obtained by solving the Euler-Lagrange equation deduced from functional 1 minimization of Eq. (2) with respect to $\Psi(\mathbf{r})$

$$
\left\{-\frac{\hbar^{2}}{2 m} \nabla^{2}+\frac{\delta \mathcal{E}_{c}}{\delta \rho}+V_{A k}\left(\left|\mathbf{r}-\mathbf{r}_{A k}\right|\right)\right\} \Psi(\mathbf{r})=\mu \Psi(\mathbf{r})
$$

where $\mu$ is the ${ }^{4} \mathrm{He}$ chemical potential for the number of He atoms in the droplet, $N \equiv \int d \mathbf{r}|\Psi(\mathbf{r})|^{2}$. In this work, this number is fixed to $N=1000$. The Ak-He potentials $\left[\gamma_{A k}\right.$ in Eqs. (2) and (3)] have been taken from Ref. 40, except for Li-He which is from Ref. 34. The lighter $\mathrm{Ak}(\mathrm{Na}$ and especially $\mathrm{Li}$ ) require a quantum mechanical treatment. This implies solving an additional Schrödinger equation for the alkali, coupled to the ${ }^{4} \mathrm{He}-$ DFT equation for helium, see Ref. 35 for more details.

The results presented in this work have been obtained using the 4He-DFT BCN-TLS computing package.[41] Details on how Eq. (2) is solved can be found in Refs. 35,42 and references therein. In short, $\Psi(\mathbf{r})$ is written on a Cartesian grid and extensive use is made of the FastFourier Transform to compute the convolutions entering the definition of $\mathcal{E}_{c}$. The droplet equilibrium configuration is obtained by imaginary-time relaxation for $\Psi(\mathbf{r})$.

Including vortices does not require further developments in the formalism. A vortex line along the $z$ axis for instance is obtained by the imprinting procedure, in which imaginary-time relaxation starts from the effective wave function[35]

$$
\Psi(\mathbf{r})=\frac{\rho_{0}^{1 / 2}(\mathbf{r})}{\sqrt{x^{2}+y^{2}}}(x+i y)
$$

where $\rho_{0}(\mathbf{r})$ is the density corresponding to a vortex-free droplet (note that in the presence of a vortex $\Psi(\mathbf{r})$ must be complex). Imaginary-time relaxation eventually converges to a configuration which is an eigenstate of the angular momentum operator $\hat{L}_{z}$ with eigenvalli $N \hbar$.

We have defined the following energies to characterize the alkali-droplet equilib;ium configurations:[35]

\begin{tabular}{|c|cc|c|cc|c|}
\hline \hline \multirow{2}{*}{$\mathrm{Ak}$} & \multicolumn{2}{|c|}{$\mathrm{Ak}^{4}{ }^{4} \mathrm{He}_{1000}$} & $S_{\mathrm{Ak}}$ & \multicolumn{2}{|c|}{$(\mathrm{Ak} @) @{ }^{4} \mathrm{He}_{1000}$} & $B_{\mathrm{Ak}}$ \\
\cline { 2 - 6 } & $E(\mathrm{~K})$ & $d(\AA)$ & $(\mathrm{K})$ & $E(\mathrm{~K})$ & $d(\AA)$ & $(\mathrm{K})$ \\
\hline \hline $\mathrm{Li}^{*}$ & -5443.32 & 27.70 & -2.53 & -5325.24 & 23.66 & 4.63 \\
$\mathrm{Na}^{*}$ & -5446.07 & 26.55 & -5.28 & -5327.30 & 23.28 & 3.94 \\
$\mathrm{~K}^{*}$ & -5447.95 & 26.83 & -7.16 & -5328.93 & 23.51 & 3.69 \\
\hline $\mathrm{Li}$ & -5450.62 & 25.06 & -9.83 & -5330.66 & 21.55 & 2.75 \\
$\mathrm{Na}$ & -5450.47 & 25.37 & -9.68 & -5330.57 & 21.99 & 2.81 \\
$\mathrm{~K}$ & -5451.08 & 26.07 & -10.29 & -5331.11 & 22.96 & 2.74 \\
$\mathrm{Rb}$ & -5451.99 & 26.06 & -11.20 & -5332.19 & 22.97 & 2.91 \\
$\mathrm{Cs}$ & -5451.29 & 26.60 & -10.50 & -5331.30 & 23.55 & 2.72 \\
\hline \hline
\end{tabular}

TABLE I: Influence of the presence of a vortex line $V$ on the characteristics of an alkali doped ${ }^{4} \mathrm{He}_{1000}$ droplet: total energy $E$, and distance $d$ of the impurity to the center of mass of the droplet. $\mathrm{Ak} @{ }^{4} \mathrm{He}_{1000}$ stands for the vortex-free droplet and $(\mathrm{Ak} @ V) @{ }^{4} \mathrm{He}_{1000}$ for the droplet hosting a linear vortex. $S_{\mathrm{Ak}}$ is the (vortex-free) impurity solvation energy defined in Eq. (5), and $B_{\mathrm{Ak}}$ is the binding energy of the impurity to the ortex line defined in Eq. (7). The results presented in this table are for the OT functional. The energy of the pure ${ }^{4} \mathrm{He}_{1000}$ droplet is $-5440.79 \mathrm{~K}$ when it is vortex-free, and $-5318.08 \mathrm{~K}$ when it hosts a linear vortex, giving $E_{V}=122.71 \mathrm{~K}$ for the energy for c-eating a vortex, Eq. (6). The asterisk in the first 3 lines of the table denotes a quantum treatment of the dopant.

Solvation energy of the alkali:

$$
S_{\mathrm{Ak}}=E\left(\mathrm{Ak} @{ }^{4} \mathrm{He}_{N}\right)-E\left({ }^{4} \mathrm{He}_{N}\right)
$$

Vortex $(V)$ energy:

$$
E_{V}=E\left(V @{ }^{4} \mathrm{He}_{N}\right)-E\left({ }^{4} \mathrm{He}_{N}\right)
$$

Binding energy of the alkali to the vortex line:

$$
\begin{aligned}
B_{\mathrm{Ak}} & =E\left[(\mathrm{Ak}+V) @{ }^{4} \mathrm{He}_{N}\right]-E\left[(\mathrm{Ak} @ V) @{ }^{4} \mathrm{He}_{N}\right] \\
& =S_{\mathrm{Ak}}+E_{V}-\left\{E\left[(\mathrm{Ak} @ V) @{ }^{4} \mathrm{He}_{N}\right]-E\left({ }^{4} \mathrm{He}_{N}\right)\right\}
\end{aligned}
$$

A negative solvation energy implies that the impurity is attached to the droplet, and a positive binding energy of the $\mathrm{Ak}$ to the vortex implies that it is attached to the vortex line. This binding energy is the result of a delicate balance between the contributing terms, and the resulting values are rather small. Intuitively, it corresponds to the kinetic energy of the superfluid flow in the volume excluded by the impurity.[7]

\section{RESULTS}

\section{A. Statics}

Figure 1 shows as an example a two-dimensional (2D) cut of the He density in a symmetry plane for a Cs-doped ${ }^{4} \mathrm{He}_{1000}$ droplet, without or with a vortex. The appearance is qualitatively the same for all the alkali atoms. 

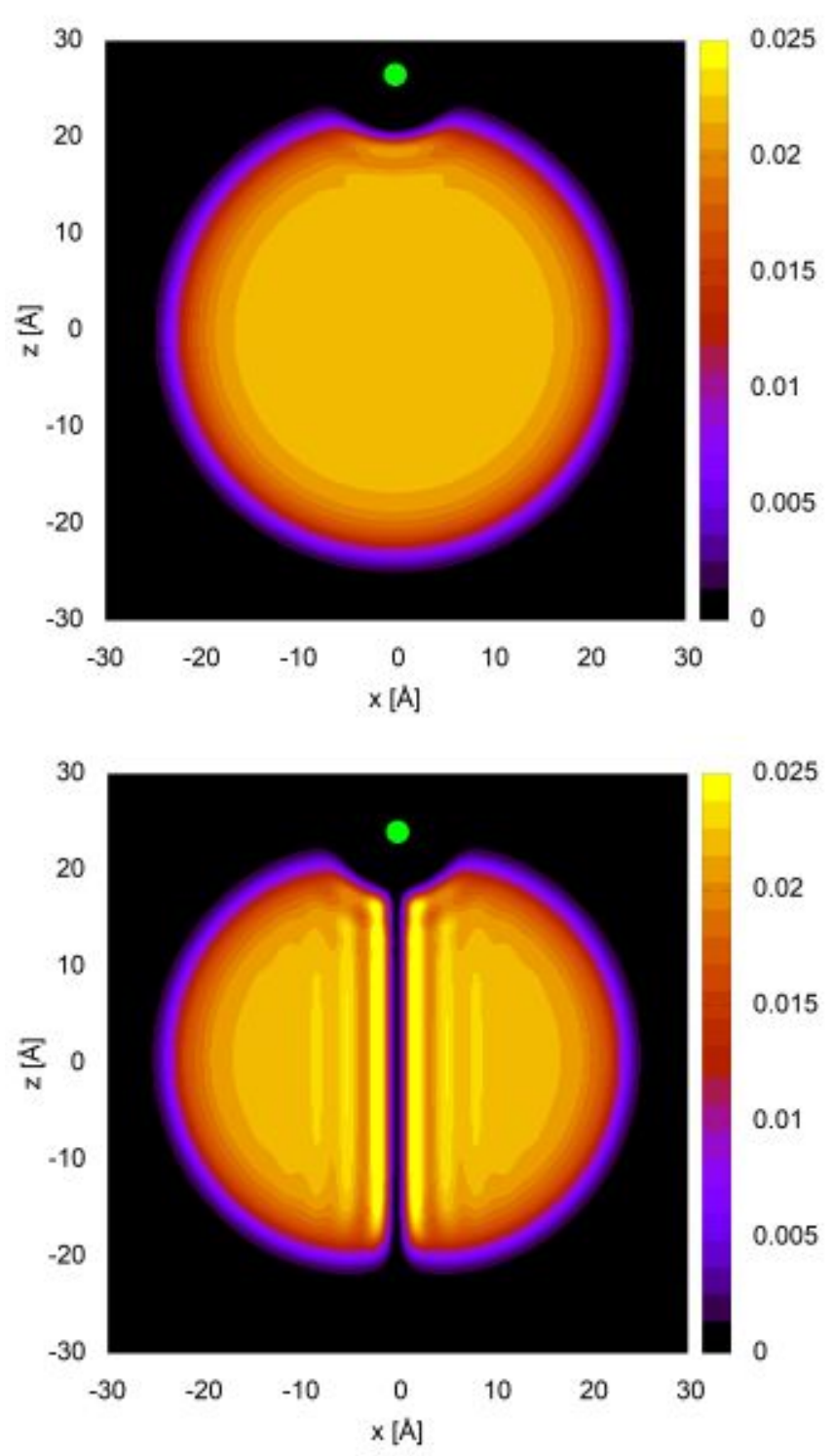

FIG. 1: 2D density in a plane of symmetry of a ${ }^{4} \mathrm{He}_{1000}$ droplet doped with a Cs atom (green dot). The color bar shows the He atom density in units of $\AA^{-3}$. Top, vortex-free droplet; bottom, vortex-hosting droplet.

The vortex is visible as an empty line along the vertical axis. Its presence makes the dopant distance $d$ to the droplet center of mass shorter, which is the results of two effects: the presence of a vortex flattens the droplet, and it makes deeper the dimple in which the alkali atom sits. This is general for all alkalis, as can be checked $n$ Table I.

Table I collects the most relevant characteristics of the calculated equilibrium configurations. As can be seen there, the binding energy to the vortex line is positive for all the alkalis, indicating that all alkali-doped vortexhosting droplets are more stable if the alkali sits at the intersection of the vortex core with the surface. The binding energy increases with atomic number, except for the heaviest one studied here, Cs, which is slightly less bound to the vortex than $\mathrm{Rb}$.

Lighter alkalis require a quantum treatment, as performed earlier in ortex-free droplets.[33, 34, 36] This was performed here for $\mathrm{Li}, \mathrm{Na}$, and $\mathrm{K}$. Because of zero-point delocalization, they sit further away from the droplet surface when described quantum mechanically. This is quite a sizeable effect, especially for $\mathrm{Li}$ as can be seen in Table I. As a consequence, the Ak solvation energy in the vortex-free droplet $\left(S_{\mathrm{Ak}}\right)$ is reduced (in absolute value), as already observed.[36] The effect becomes smaller with increasing atomic number, as expected. The binding energy to the vortex line $\left(B_{\mathrm{Ak}}\right)$ increases in the quantum treatment, likely because the excluded volume already mentioned[7] increases due to zero point delocalization of the impurity.

As indicated before, we have also used the solid functional for this study. The resulting impurity distances to the droplet center of mass, solvation and vortex-binding energies differ only very slightly from the OT ones, as can be checked in Table I in the Supplementary Material, and the qualitative conclusions are not affected.

\section{B. Absorption spectra}

We now turn to the influence of a vortex on the absorption spectrum of an alkali-doped helium droplet. Once the equilibrium configuration of the system has been determined, the absorption spectrum is calculated as explained in Ref. 17. The required excited $\Pi$ and $\Sigma \mathrm{Ak}-\mathrm{He}$ pair potentials were taken from Pascale,[43] except in the case of $\mathrm{Li}$, for which we have used those from Ref. 34 . The method, semiclassical in nature, essentially yields the peak energies of the absorption spectrum and their relative intensity.

The width of the spectrum comes from fluctuations in the helium density and/or, to a lesser extent, from the probability distribution given by the square of the $\mathrm{Ak}$ wave function when it is treated quantum mechanically. To incorporate the helium density fluctuations, we use the DF sampling method described in detail in Refs. 42 and 44. The additional spectral width originating from the dopant zero-point delocalization was negligible in comparison and was not calculated.

\section{1. $n p \leftarrow n s$ absorption spectra}

Figure 2 shows the effect of a vortex on the $n p \leftarrow n s$ absorption spectrum of the alkali atoms attached to a ${ }^{4} \mathrm{He}_{1000}$ droplet. It can be seen that the absorption peaks are further blue-shifted and broadened in the presence of a vortex line. In addition, treating the dopant quantum mechanically decreases the shift and the width as expected, since the impurity is on average further away from the droplet due to its zero point motion. This is illustrated in the figure for the case of $\mathrm{Na}$ and also happens 

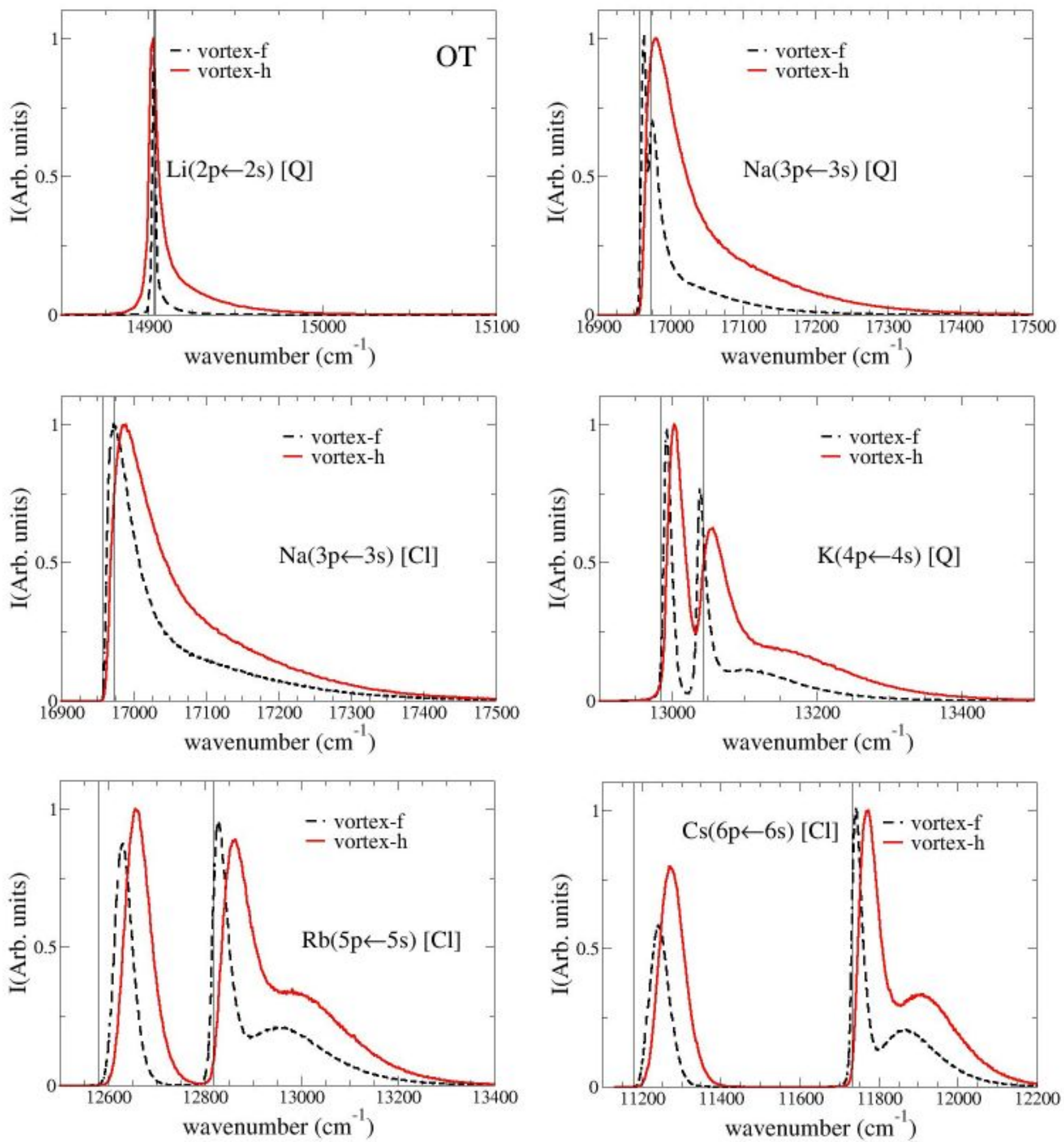

FIG. 2: $\quad n p \leftarrow n s$ absorption spectrum for the alkali-doped ${ }^{4} \mathrm{He}_{1000}$ droplet obtained with the OT functional. Li, Na, and $\mathrm{K}$ atoms are treated quantum-mechanically $([\mathrm{Q}])$, whereas $\mathrm{Rb}$, and $\mathrm{Cs}$ are treated classically $([\mathrm{Cl}])$. For the sake of comparison, the results for Na obtained with the classical description are also shown. Black dashed line: vortex-free (vortex-f) droplet; red solid line: vortex-hosting (vortex-h) droplet. Gas-phase atomic transitions are represented by thin vertical lines. Units are arbitrary for absorption intensity, and $\mathrm{cm}^{-1}$ for excitation energy. 
for Li and $\mathrm{K}$. The quantitative analysis of the corresponding spectral characteristics s presented in Table II.

Let us mention that the semiclassical approach is less justified in the case of $\mathrm{Li}$, for which the absorption spectrum is dominated by bound-bound transitions. [27, 34] Including them properly would require e.g. to carry out a Fourier analysis of the time-correlation function of the $\mathrm{Li}$ atom moving in the full $3 \mathrm{D}^{2} \Pi_{1 / 2},{ }^{2} \Pi_{3 / 2}$, and ${ }^{2} \Sigma_{1 / 2}$ potentials. [34] Hence the corresponding spectral characteristics are omitted in Table II. The spectrum obtained semiclassically is till presented in Fig. 2 for the sake of completeness.

It is tempting to make a direct comparison with experimental spectra which have been measured for all the calculated transitions. However, quantitative agreement of peak positions and line shapes from the DFT as well as the quality of available interaction potentials is very limited and deviations from measured absorption lines are beyond the vortex-induced differences calculated in this work. In particular, shifts due to the presence of a vortex are significantly smaller than the widths of the peaks. This is all the more clear when examining Table III, where the difference $\Delta$ between the shift with and without vortex is compared to the average FWHM. For all cases studied here, the average width is of the order of twice the difference in shifts. Having said this, comparing line positions and shapes is not suited to experimentally asses the presence of vortices for these transitions. Moreover, for very large droplets, coherent diffraction imaging results suggest that the proportion of droplets hosting a vortex is only on the or der of a few percent, [20-22] implying that changes in the overall peak shape are at most on the same level. Although experimental spectra can readily be obtained on that level of signal-to-noise ratios, only methods being able to detect differences between vortexfree and vortex-hosting droplets would contain valuable information. As long as there are no methods at hand to specifically control the content of vortices of produced droplets in spectroscopic measurements, spectral changes will remain concealed.

\section{2. $(n+1) s \leftarrow n s$ or $(n+1) p \leftarrow n s$ absorption spectra}

The spectral characteristics of alkali-doped helium droplets are mostly governed by the usually repulsive interaction of their excited state orbital with the surrounding helium at the equilibrium configuration of the ground state. Since this orbital is more diffuse and more extended in higher $\left(n^{\prime}>n\right)$ electronic states, its sensitivity to changes in the surrounding helium density is increased. Hence, absorption spectra to $n^{\prime}>n$ excited states could reveal more differences between vortexhosting and vortex-free droplets than $n p \leftarrow n s$ ones.

We have tested this idea on three cases which have already been studied before in vortex-free droplets, namely $\mathrm{K}(5 s \leftarrow 4 s), \operatorname{Rb}(6 p \leftarrow 5 s)$, and $\operatorname{Cs}(7 s \leftarrow 6 s)$ absorption $\square$ spectra $[32,36,45,46]$ Fig. 3 shows the corresponding
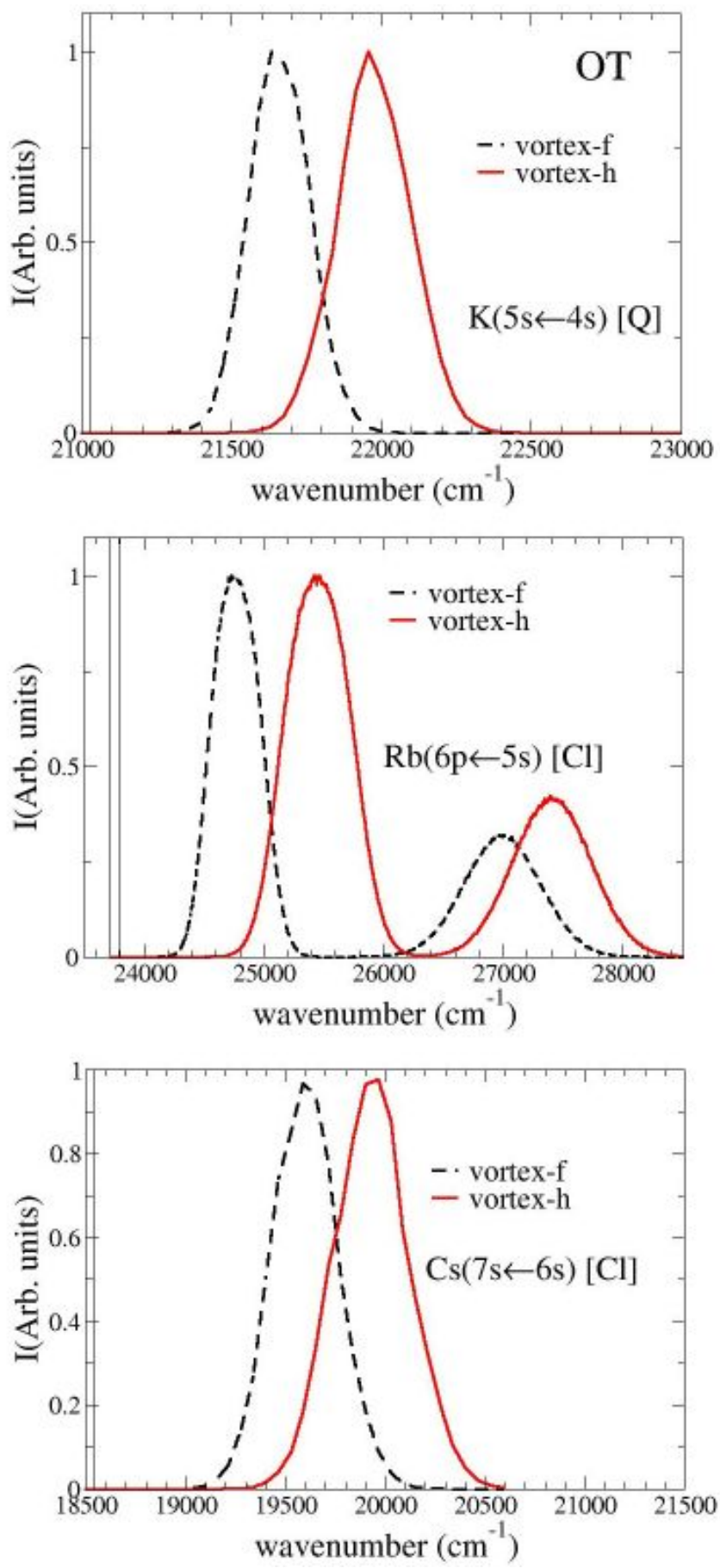

FIG. 3: Dipole absorption spectrum for $\mathrm{K}(5 s \leftarrow 4 s)$ (top), $\mathrm{Rb}(6 p \leftarrow 5 s)$ (middle), and $\operatorname{Cs}(7 s \leftarrow 6 s)$ (bottom) transitions in a vortex-free (black dashed line) or vortex-hosting (red solid line) ${ }^{4} \mathrm{He}_{1000}$ droplet obtained with the OT functional. The $\mathrm{K}$ atom is treated quantum mechanically and the $\mathrm{Rb}$ and Cs atoms classically. The thin vertical lines represent gasphase atomic transitions. Units are arbitrary for absorption intensity, and $\mathrm{cm}^{-1}$ for excitation energy. 


\begin{tabular}{|c|c|c|c|c|c|c|c|c|c|c|c|c|}
\hline \multirow{3}{*}{$\mathrm{Ak}$} & \multirow{2}{*}{\multicolumn{3}{|c|}{$\begin{array}{c}(\Delta E)_{\text {vortex }-\mathrm{f}} \\
\left(\mathrm{cm}^{-1}\right)\end{array}$}} & \multirow{2}{*}{\multicolumn{3}{|c|}{$\begin{array}{c}(\Delta E)_{\text {vortex }-\mathrm{h}} \\
\left(\mathrm{cm}^{-1}\right)\end{array}$}} & \multicolumn{6}{|c|}{ FWHM $\left(\mathrm{cm}^{-1}\right)$} \\
\hline & & & & & & & \multirow{2}{*}{\multicolumn{2}{|c|}{\begin{tabular}{|c|}
${ }^{2} \Pi_{1 / 2}$ \\
vortex-f vortex-h \\
\end{tabular}}} & \multirow{2}{*}{\multicolumn{2}{|c|}{\begin{tabular}{|c|}
${ }^{2} \Pi_{3 / 2}$ \\
vortex-f vortex-h
\end{tabular}}} & \multirow{2}{*}{\multicolumn{2}{|c|}{\begin{tabular}{|c|}
${ }^{2} \Sigma_{1 / 2}$ \\
vortex-f vortex-h
\end{tabular}}} \\
\hline & ${ }^{2} \Pi_{1 / 2}$ & ${ }^{2} \Pi_{3 / 2}$ & ${ }^{2} \Sigma_{1 / 2}$ & ${ }^{2} \Pi_{1 / 2}$ & ${ }^{2} \Pi_{3 / 2}$ & ${ }^{2} \Sigma_{1 / 2}$ & & & & & & \\
\hline $\mathrm{Na}^{*}(3 p \leftarrow 3 s)$ & 6.4 & 3.1 & 58.3 & 12.9 & 18.5 & 76.5 & 10 & 21 & 18 & 43 & 111 & 132 \\
\hline $\mathrm{K}^{*}(4 p \leftarrow 4 s)$ & 7.6 & -4.5 & 66.4 & 14.8 & 7.0 & 97.8 & 13 & 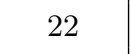 & 14 & 34 & 120 & 135 \\
\hline $\mathrm{Na}(3 p \leftarrow 3 s)$ & 17.4 & 29.9 & 140.0 & 33.0 & 65.9 & 171.1 & 2 & 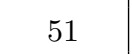 & 63 & 99 & 204 & 212 \\
\hline $\mathrm{K}(4 p \leftarrow 4 s)$ & 13.4 & 3.9 & 116.8 & 25.1 & 26.7 & 151.2 & 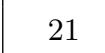 & $\pi$ & 3 & 62 & 178 & 185 \\
\hline $\mathrm{Rb}(5 p \leftarrow 5 s)$ & 51.2 & 11.0 & 138.6 & 74.0 & 37.1 & 169.7 & 43 & 59 & 37 & 63 & 176 & 185 \\
\hline Cs $(6 p \leftarrow 6 s)$ & 62.6 & 9.5 & 131.3 & 88.9 & 34.0 & $168.5 \mathrm{~d}$ & 57 & 71 & 31 & 53 & 158 & 167 \\
\hline $\mathrm{K}^{*}(5 s \leftarrow 4 s)$ & - & - & 618.9 & - & 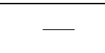 & 880.6 & 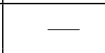 & - & - & - & 242 & 270 \\
\hline $\mathrm{K}(5 s \leftarrow 4 s)$ & - & - & 839.4 & - & - & 1079.3 & - & - & - & - & 282 & 307 \\
\hline $\operatorname{Rb}(6 p \leftarrow 5 s)$ & 942.6 & 1101.3 & 3205.7 & 1518.0 & 1766.8 & 3535.6 & 314 & 421 & 326 & 428 & 743 & 761 \\
\hline $\mathrm{Cs}(7 s \leftarrow 6 s)$ & - & - & 1070.3 & - & - & 1402.1 & - & - & - & - & 384 & 434 \\
\hline
\end{tabular}

TABLE II: Comparison of the $n p \leftarrow n s$ spectral characteristics of an alkali atom sitting on a vortex-free (vortex-f) and vortexhosting (vortex-h) ${ }^{4} \mathrm{He}_{1000}$ nanodroplet. $2^{\text {nd }}$ to $4^{\text {th }}$ columns: energy shifts $(\Delta E)_{\text {vortex-f }}$ for the vortex-free droplet with respect to the gas-phase atomic lines, calculated from the absorption maxima for the ${ }^{2} \Pi_{1 / 2},{ }^{2} \Pi_{3 / 2}$, and ${ }^{2} \Sigma_{1 / 2}$ final states; $5^{\text {th }}$ to $7^{\text {th }}$ columns: energy shifts $(\Delta E)_{\text {vortex }-\mathrm{h}}$ for the vortex-hosting droplet; last 6 columns: full width at half maximum (FWHM) for each peak for the vortex-free or vortex-hosting droplet. The top part of the table is for $n p \leftarrow n s$ absorption spectra and the bottom part, separated by a horizontal line, is for $(n+1) s \leftarrow n s$ or $(n+1) p \leftarrow n s$ absorption spectra. The asterisk denotes a quantum treatment of the dopant. 
absorption spectra, and the shifts and FWHM are collected in the lower part of Table II. As expected, the difference in shifts between spectra originating from vortexhosting and vortex-free droplets are much larger than in the $n p \leftarrow n s$ spectra. They are now of the same order of magnitude as the spectral widths, as can be seen more clearly in Table III. This could make them distinguishable in the most favorable cases. Yet, several groups have examined $(n+1) \leftarrow n$ and higher transitions and did not report any unassigned shoulder or separated peak.[2932] This is not surprising, providing, as discussed above, an expected contribution of vortices only on the percent level. Since the peak shapes and consequently also the far-out wings cannot be reproduced by the simulations on that level of accuracy, without the ability of measuring difference spectra it will be very difficult to confirm vortices in spectroscopic measurements also at these transitions.

Checking which case would be the most favorable one for vortex-line detection would require a systematic search for all the alkalis and their $(n+1) \leftarrow n$ or even $(n+2) \leftarrow n$ transitions, which in turn would call for systematic improvement of the commonly used Pascale's helium-alkali interactions in the excited electronic states; for instance, the He-Rb*(6p) interactions are notoriously too repulsive, resulting in too large spectral shifts. This systematic study is beyond the scope of the present work.

\section{SUMMARY AND OUTLOOK $\square \square$}

We have investigated the possibility of using alkali absorption spectroscopy as a means of detecting the presence of vortices in a superfluid helium droplet. To this end, we have conducted ${ }^{4} \mathrm{He}-\mathrm{DFT}$ simulations using the most accurate functional to date, namely the OT functional. For the sake of completeness, the absorption spectra of Figs. 2 and 3 obtained using the solid functional of Ref. 39, which lacks the backflow and non-local ki-

\begin{tabular}{|c|cc|cc|cc|}
\hline \hline \multirow{2}{*}{$\mathrm{Ak}$} & \multicolumn{2}{|c|}{${ }^{2} \Pi_{1 / 2}$} & \multicolumn{2}{|c|}{${ }^{2} \Pi_{3 / 2}$} & \multicolumn{2}{|c|}{${ }^{2} \Sigma_{1 / 2}$} \\
\cline { 2 - 7 } & $\begin{array}{c}\Delta \\
\left(\mathrm{cm}^{-1}\right)\end{array}$ & $\begin{array}{c}\left.\mathrm{cm}^{-1}\right) \\
\left(\mathrm{cm}^{-1}\right)\end{array}$ & $\begin{array}{c}\left.\mathrm{cm}^{-1}\right) \\
\left(\mathrm{cm}^{-1}\right)\end{array}$ & \begin{tabular}{c}
$\left.\mathrm{cm}^{-1}\right)$ \\
\hline
\end{tabular} \\
\hline \hline $\mathrm{Na}^{*}(3 p \leftarrow 3 s)$ & 6.4 & 15.5 & 15.5 & 30.5 & 18.2 & 121.5 \\
$\mathrm{~K}^{*}(4 p \leftarrow 4 s)$ & 7.2 & 17.5 & 11.5 & 24 & 31.4 & 127.5 \\
$\mathrm{Na}(3 p \leftarrow 3 s)$ & 15.7 & 39.5 & 36.0 & 81 & 31.1 & 208 \\
$\mathrm{~K}(4 p \leftarrow 4 s)$ & 11.7 & 28.5 & 22.8 & 48 & 34.4 & 181.5 \\
$\mathrm{Rb}(5 p \leftarrow 5 s)$ & 22.8 & 51 & 26.0 & 50 & 31.1 & 180.5 \\
$\mathrm{Cs}(6 p \leftarrow 6 s)$ & 26.3 & 74 & 24.5 & 42 & 37.2 & 162.5 \\
\hline $\mathrm{K}(5 s \leftarrow 4 s)$ & - & - & - & - & 262 & 256 \\
$\mathrm{~K}(5 s \leftarrow 4 s)$ & - & - & - & - & 240.0 & 294.5 \\
$\mathrm{Rb}(6 p \leftarrow 5 s)$ & 575.3 & 367.5 & 665.5 & 377 & 329.9 & 752 \\
$\mathrm{Cs}(7 s \leftarrow 6 s)$ & - & - & - & - & 331.8 & 409 \\
\hline \hline
\end{tabular}

TABLE III: Comparison of the energy shift difference $\Delta$ between the vortex-hosting and vortex-free droplet and $\Gamma$, the half sum of the FWHM of the spectra with or without ortex, calcu ated from data in Table II. netic energy terms, are presented in the Supplementary Material, together with the corresponding table of their spectral characteristics.

The results show a blue shift and broadening of the absorption peaks in a vortex-hosting compared to a vortexfree droplet. However, these spectral modifications are very modest for $n p \leftarrow n s$ excitation: the shifts are significantly smaller than the peak widths. Since the proportion of droplets hosting a vortex is expected to be small, these modifications will be difficult to extract from experimental observations. In addition, the experimental droplet size distribution also affects the spectrum, which would make the identification of vortex presence even more difficult.

The situation is more favorable when turning to higher $n^{\prime} p \leftarrow n s$ or $n^{\prime} s \leftarrow n s\left(n^{\prime}>n\right)$ excitations. In this case the excited state orbital of the alkali atom is more diffuse and more extended, which makes it more sensitive to changes in its local environment. As a consequence, the corresponding $n^{\prime} p$ or $n^{\prime} s \leftarrow n s$ absorption spectra are more clearly affected, with larger blue shifts and broadening of the peaks, the shifts being then of the same order of magnitude as the widths. This could make experimental detection of vortex-hosting droplets feasible, at least if one finds ways to increase their proportion.

Note that in previous works simulating the capture of dopants by vortex-hosting droplets, dopants were found to spin around vortex lines rather than being attached to them. [26, 47, 48] Spectral modifications in the alkali absorption spectrum would be smaller in that case. However, these simulations were describing the first several hundred picoseconds of the capture dynamics. The alkali location used in the current work is that corresponding to its equilibrium location, i.e., at the vortex line-droplet surface intersection. The flight time of the droplet between the pickup cell and detection is of the order of millisecond, which gives plenty of time for the system to relax after the capture process and to reach its equilibrium configuration.

In droplets formed by supersonic expansion of helium gas into vacuum, vortex nucleation is expected to be scarce. When occurring it is due to collisions with impurities, as observed in studies of impurity capture by b@ium nanodroplets. $[23,24]$ The faster the impurity, the more probable vortex creation is. On the other hand, if the impurity is too fast it can escape the droplet, even if the He-impurity interaction is attractive. Combining these arguments, one could imagine a two-step experiment where the amount of vortices could be changed: collision with fast heliophilic impurities to create vortices, then usual pickup of alkalis for detection through $n^{\prime} s \leftarrow n s$ or $n^{\prime} p \leftarrow n s\left(n^{\prime} \geq n\right)$ absorption spectroscopy. Additional doping from the first step could be discriminated by spectroscopy. Certainly, the first step also induces changes in the droplet size distribution. However, corresponding effects are reasonably understood and can be modelled.[49] Moreover, cluster size dependencies of electronic spectra of alkalis appear to be weak beyond a 
few thousand helium atoms per droplet and can be accurately characterized.[50]

Direct diffractive imaging of small droplets being impossible at present, the ultimate detection of vortices in helium droplets made of a few thousand atoms will be likely based on finding an observable sensitive to the changes caused by vortices in the impurity environment, that might be simultaneously studied experimentally and theoretically. Let us point out that femtosecond pump-probe photoionization of alkali atoms attached to helium droplets provides such observables, as e.g. desorption/fall-back times and final velocities of desorbed atoms and ions.[45] However, it does not seem that these observables will be more sensitive to the presence of vortices than the alkali absorption spectrum.

\section{SUPPLEMENTARY MATERIAL}

See supplementary material for the results obtained with the solid derisity functional of Ref. 39 .

\section{Acknowledgments}

This work has been performed under Grant No FIS2017-87801-P (AEI/FEDER, UE). M.B. and E.G.A. thank the Université Fédérale Toulouse MidiPyrénées for financial support throughout the "Chaires d'Attractivité 2014" Programme IMDYNHE. A computer grant from CALMIP high performance computer center (grant P1039) is gratefully acknowledged.

\section{Data availability statement}

The data that support the findings of this study are available from the corresponding author upon reasonable request.
[1] J. P. Toennies and A. F. Vilesov, Angew. Chem. Phys. 43, 2622 (2004).

[2] K. K. Lehmann and G. Scoles, Science 279, 2065 (1998).

[3] F. Stienkemeier and K. K. Lehmann, J. Phys. B 39, R127 (2006).

[4] M. Y. Choi, G. E. Douberly, T. M. Falconer, W. K. Lewis, C. M. Lindsay, J. M. Merritt, P. L. Stiles, and R. E. Miller, Int. Rev. Phys. Chem. 25, 15 (2006).

[5] J. Tiggesbäumker and F. Stienkemeier, Phys. Chem. Chem. Phys. 9, 4748 (2007).

[6] C. Callegari and W. E. Ernst, in Handbook of High Resolution Spectroscopy, Vol. 3 (Whiley, New York, 2011), p. 1551.

[7] R. J. Donnelly, Quantized vortices in helium II, Cambridge Studies in Low Temperature Physics, Vol. 3 (Cambridge University Press, Cambridge, U.K., 1991).

[8] F. Ancilotto, M. Pi, and M. Barranco, Phys. Rev. B 97, 184515 (2018).

[9] O. Gessner and A. F. Vilesov, Annu. Rev. Phys. Chem. 70, 173 (2019).

[10] A. Fetter, Rev. Mod. Phys. 81, 647 (2009).

[11] H. Saarikoski, S. M. Reimann, A. Harju, and M. Manninen, Rev. Mod. Phys. 82, 2785 (2010).

[12] M. Barranco, R. Guardiola, S. Hernández, R. Mayol, J. Navarro, and M. Pi, J. Low Temp. Phys. 142, 1 (2006).

[13] E. Sola, J. Casulleras, and J. Boronat, Phys. Rev. B 76, 052507 (2007).

[14] J. D. Close, F. Federman, K. Hoffmann, and N. Quaas, J. Low Temp. Phys. 111, 661 (1998).

[15] D. Mateo, A. Hernando, M. Barranco, and M. Pi, J. Low Temp. Phys. 158, 397 (2010)

[16] F. Ancilotto, M. Barranco, and M. Pi, Phys. Rev. Lett. 91,105302 (2003).

[17] A. Hernando, M. Barranco, R. Mayol, M. Pi, and M. Krośnicki, Phys. Rev. B 77, 024513 (2008).

[18] F. Stienkemeier, F. Meier, and H. O. Lutz, J. Chem.
Phys. 107, 10816 (1997).

[19] R. M. Tanyag, C. F. Jones, C. Bernando, S. M. O'Connell, D. Verma, and A. F. Vilesov, in Theoretical and Computational Chemistry Series No. 11, O. Dulieu and A. Osterwalder editors. Royal Society of Chemistry, Cambridge, p. 389 (2018).

[20] L. F. Gomez, K. R. Ferguson, J. P. Cryan, C. Bacellar, R. M. P. Tanyag, C. Jones, S. Schorb, D. Anielski, A. Belkacem, C. Bernando, R. Boll, J. Bozek, S. Carron, G. Chen, T. Delmas, L. Englert, S. W. Epp, B. Erk. L. Foucar, R. Hartmann, A. Hexemer, M. Huth, J. Kwok, S. R. Leone, J. H. S. Ma, F. R. N. C. Maia, E. Malmerberg, S. Marchesini, D. M. Neumark, B. Poon, J. Prell, D. Rolles, B. Rudek, A. Rudenko, M. Seifrid, K. R. Siefermann, F. P. Sturm, M. Swiggers, J. Ullrich, F. Weise, P. Zwart, C. Bostedt, O. Gessner, and A. F. Vilesov, Science 345, 906 (2014)

[21] B. Langbehn, K. Sander, Y. Ovcharenko, C. Peltz, A. Clark, M. Coreno, R. Cucini, M. Drabbels, P. Finetti, M. Di Fraia, L. Giannessi, C. Grazioli, D. Iablonskyi, A. C. LaForge, T. Nishiyama, V. Oliver Álvarez de Lara, P. Piseri, O. Plekan, K. Ueda, J. Zimmermann, K. C. Prince, F. Stienkemeier, C. Callegari, T. Fennel, D. Rupp, and T. Möller, Phys. Rev. Lett. 121, 255301 (2018).

[22] S. M. O. O'Connell, R. M. P. Tanyag, D. Verma, Ch. Bernando, W. Pang, C. Bacellar, C. A. Saladrigas, J. Mahl, B. W. Toulson, Y. Kumagai, P. Walter, F. Ancilotto, M. Barranco, M. Pi, Ch. Bostedt, O. Gessner, and A. F. Vilesov, arXiv:1910.12926, to be published in Phys. Rev. Lett. (2020).

[23] A. Leal, D. Mateo, A. Hernando, M. Pi, and M. Barranco, Phys. Chem. Chem. Phys. 16, 23206 (2014).

[24] D. Mateo, A. Leal, A. Hernando, M. Barranco, M. Pi, F. Cargnoni, M. Mella, X. Zhang, and M. Drabbels, J. Chem. Phys. 140, 131101 (2014).

[25] A. Leal, D. Mateo, A. Hernando, M. Pi, M. Barranco, A. 
Ponti, F. Cargnoni, and M. Drabbels, Phys. Rev. B 90, 224518 (2014)

[26] F. Coppens, F. Ancilotto, M. Barranco, N. Halberstadt, and M. Pi, Phys. Chem. Chem. Phys. 19, 24805 (2017).

[27] F. Stienkemeier, J. Higgins, C. Callegari, S. I. Kanorsky, W. E. Ernst, and G. Scoles, Z. Phys. D 38, 253 (1996).

[28] O. Bünermann, G. Droppelmann, A. Hernando, R. Mayol, and F. Stienkemeier, J. Phys. Chem. A 111, 12684 (2007).

[29] A. Pifrader, O. Allard, G. Auböck, C. Callegari, W. E. Ernst, R. Hubert, and F. Ancilotto, J. Chem. Phys. 133 $164502(2010)$.

[30] F. Lackner, G. Krois, M. Theisen, M. Koch, and W. E. Ernst, Phys. Chem. Chem. Phys. 13, 18781 (2011).

[31] E. Loginov, C. Callegari, F. Ancilotto, and M. Drabbels, J. Phys. Chem. A 115, 6779 (2011).

[32] L. Fechner, B. Grüner, A. Sieg, C. Callegari, F. Ancilotto, F. Stienkemeier, and M. Mudrich, Phys. Chem. Chem. Phys. 14, 3843 (2012)

[33] A. Hernando, M. Barranco, R. Mayol, M. Pi, F. Ancilotto, O. Bünermann, and F. Stienkemeier, J. Low Temp. Phys. 158, 105 (2010).

[34] A. Hernando, R. Mayol, M. Pi, M. Barranco, I. S. K. Kerkines, and A. Mavridis, Int. J. of Quantum Chem. 111, 400 (2011).

[35] F. Ancilotto, M. Barranco, F. Coppens, J. Eloranta, N. Halberstadt, A. Hernando, D. Mateo, and M. Pi, Int. Rev. Phys. Chem. 36, 621 (2017).

[36] M. Martinez, F. Coppens, M. Barranco, N. Halberstadt, and M. Pi, Phys. Chem. Chem. Phys. 21, 3626 (2019).

[37] F. Coppens, J. von Vangerow, A. Leal, M. Barranco, N. Halberstadt, M. Mudrich, M. Pi, and F. Stienkemeier,
Eur. Phys. J. D 73:94 (2019)

[38] F. Dalfovo, A. Lastri, L. Pricaupenko, S. Stringari, and J. Treiner, Phys. Rev. B 52, 1193 (1995).

[39] F. Ancilotto, M. Barranco, F. Caupin, R. Mayol, and M. Pi, Phys. Rev. B 72, 214522 (2005).

[40] S. H. Patil, J. Chem. Phys. 94, 8089 (1991).

[41] M. Pi, F. Ancilotto, F. Coppens, N. Halberstadt, A. Hernando, A. Leal, D. Mateo, R. Mayol, and M. Barranco, 4He-DFT BCN-TLS: A Computer Package for Simulating Structural Properties and Dynamics of Doped Liquid Helium-4 Systems. https://github.com/bcntls2016/

[42] M. Barranco, F. Coppens, N. Halberstadt, A. Hernando, A. Leal, D. Mateo, R. Mayol, and M. Pi, Zero temperature DFT and TDDFT for ${ }^{4} \mathrm{He}$ : A short guide for practitioners. https://github.com/bcntls2016/

[43] J. Pascale, Phys. Rev. A 28, 632 (1983).

[44] D. Mateo, A. Hernando, M. Barranco, R. Mayol, and M. Pi, Phys. Rev. B 83, 174505 (2011).

[45] J. von Vangerow, F. Coppens, A. Leal, M. Pi, M. Barranco, N. Halberstadt, F. Stienkemeier, and M. Mudrich, J. Phys. Chem. Lett. 8, 307 (2017).

[46] J. von Vangerow, A. Stieg, F. Stienkemeier, M. Mudrich, A. Leal, D. Mateo, A. Hernando, M. Barranco, and M. Pi, J. Phys. Chem. A 118, 6604 (2014).

[47] F. Coppens, F. Ancilotto, M. Barranco, N. Halberstadt, and M. Pi, Phys. Chem. Chem. Phys. 21, 17423 (2019).

[48] I. A. Pshenichnyuk and N. G. Berloff, Phys. Rev. B 94, 184505 (2016).

[49] O. Bünermann and F. Stienkemeier, Eur. Phys. J. D 61 , 645 (2011).

[50] F. Stienkemeier, J. Higgins, W. E. Ernst, and G. Scoles, Z. Phys. B 98, 413 (1995). 

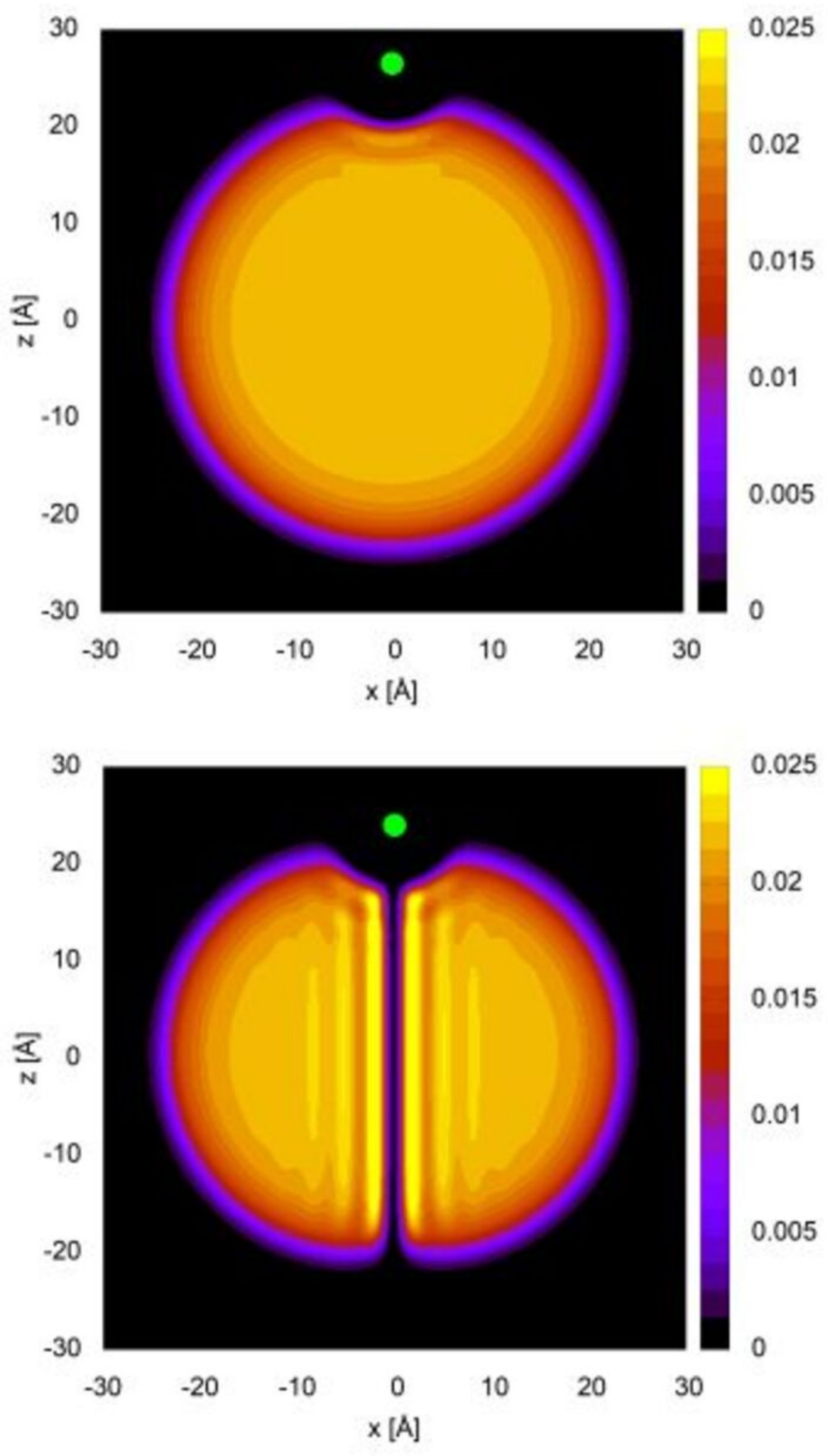
